\title{
DISCUSSIONS ON THE CANCELLATION EFFECT ON A CIRCULAR ORBIT*
}

\author{
R. Li and Ya. S. Derbenev, Jefferson Lab, 12000 Jefferson Ave., Newport News, VA 23606, USA
}

\section{INTRODUCTION}

As reviewed in Ref. [1] and [2], the existence of the transverse Lorentz force induced by radial acceleration of the particles on a circular orbit was first pointed out by Talman [3]. For a line bunch (with zero transverse extent), this force displays logarithmic divergence due to local contributions, namely, the singular contribution of the nearby particle interaction. For a bunch with finite transverse size, this logarithmic divergence is removed; however, the local contributions cause the curvature-induced transverse collective force to have sensitive dependence on the particle's transverse offset from the design orbit, as depicted in Fig. 1 of Ref. [3]. Later Lee pointed out [4] that for a line coasting beam, the effect of the logarithmic divergence of the curvature-induced transverse collective force is largely cancelled by the effect of the change of kinetic energy (induced by the same transverse collective force for the coasting beam case). Some following studies $[5,6,7,2]$ conclude that this cancellation, discussed earlier for a coasting beam, also applies for general bunched beam under relativistic motion on a curved orbit. As summarized in Ref. [1], the transverse dynamics of a test particle in the electron bunch is determined by (1) the radial collective force $F_{\perp}(t)$ and (2) the deviation of the kinetic energy from the design energy $\Delta E(t)=E-E_{0}$ :

$\frac{d}{d t}\left(\gamma m \frac{d r}{d t}\right)-\gamma_{0} m c^{2}\left(\frac{\beta_{s}^{2}}{r}-\frac{\beta_{s} \beta_{0}}{R}\right)=F_{\perp}(t)+\frac{\beta_{s}^{2} \Delta E(t)}{r}$

where $r=R(s)+x$,

$$
\begin{gathered}
\Delta E=\left[\gamma(0) m c^{2}+e \Phi(0)\right]-e \Phi(t)+e \int_{0}^{t} \frac{\partial(\Phi-\boldsymbol{\beta} \cdot \mathbf{A})}{\partial t^{\prime}} d t^{\prime}, \\
F_{\perp}=-e \frac{\partial(\Phi-\boldsymbol{\beta} \cdot \mathbf{A})}{\partial r}-e \frac{d A_{r}}{c d t}+F^{\mathrm{CSCF}} .
\end{gathered}
$$

Here the third term in Eq. (3) is denoted as centrifugal space charge force:

$$
F^{\mathrm{CSCF}}=e \beta_{s} A_{s} / r .
$$

It turns out that for the two driving terms in Eq. (1), the effect of the $F^{\mathrm{CSCF}}$ term in Eq. (3) is largely cancelled by the effect of the $-e \Phi(t)$ term in $\Delta E$ of Eq. (2). This is shown by rearranging terms in Eq. (1):

$\frac{d}{d t}\left(\gamma m \frac{d r}{d t}\right)-\gamma_{0} m c^{2}\left(\frac{\beta_{s}^{2}}{r}-\frac{\beta_{s} \beta_{0}}{R}\right)=G_{0}+G_{v}+G_{r}+G_{c}$

with

\footnotetext{
* Work supported by the U.S. Dept. of Energy under Contract No.DEAC05-84ER40150
}

$$
\begin{aligned}
G_{0} & =\beta_{s}^{2} \frac{\left[\gamma(0) m c^{2}+e \Phi(0)\right]-\gamma_{0} m c^{2}}{r} \\
G_{v} & =e \frac{\beta_{s}^{2}}{r} \int_{0}^{t} \frac{\partial(\Phi-\boldsymbol{\beta} \cdot \mathbf{A})}{\partial t^{\prime}} d t^{\prime}, \\
G_{r} & =F_{r}^{\mathrm{eff}} \equiv-e \frac{\partial(\Phi-\boldsymbol{\beta} \cdot \mathbf{A})}{\partial r}-e \frac{d A_{r}}{c d t}, \\
G_{c} & =F^{\mathrm{CSCF}}-e \beta_{s}^{2} \frac{\Phi(t)}{r}=e \beta_{s} \frac{A_{s}(t)-\beta_{s} \Phi(t)}{r} .
\end{aligned}
$$

The essence of the cancellation effect is that in Eq. (9) both $A_{s}(t)$ and $\Phi(t)$ are dominated by the same local contributions (see details in Discussions), hence they are well cancelled in $G_{c}$, as discussed analytically in Sec. 3.2.2 of Ref. [2] and as shown by numerical results demonstrated in Ref. [7]. As discussed earlier [2, 7], $G_{v}$ and $G_{r}$ are related to effective forces which are majorly contributed by nonlocal interactions, and $G_{0}$ represents the effect of the initial energy condition of the test particle.

Recently, controversies of the cancellation effect are brought up in Ref. [1], and possible misconceptions are pointed out. In the following, we list several points raised in Ref. [1] concerning the curvature-induced transverse force and the cancellation effect. Since these are indeed points that easily cause confusion, we seek to clarify them through discussions based on the above basic equations, and show how they can be perceived from the viewpoint of the cancellation picture.

\section{DISCUSSIONS}

1. The logarithmic singularity in $F_{\perp}$ comes from the choice of particular density distributions. It can be removed for a bunch with finite transverse size.

This is a true statement. However, even though the singularity is removed for a finite bunch, the mechanism which causes the logarithmic divergence of $F_{\perp}$ for a line bunch, namely, the local contribution by the nearby particle interaction (see item 4 ), still causes the sensitive dependence of $F_{\perp}$ on the particle's transverse offset. This transverse sensitivity of $F_{\perp}$ for a finite size beam, as shown in Fig. 1 of Ref. [3] or Fig. 1 of Ref. [7], is the subject of concerns for its possible deteriorating effect on particles' transverse dynamics in storage rings [3] or in a bunch compression chicane. The cancellation effect mainly refers to the cancellation of this singular contribution to $F_{\perp}$ by nearby particle interaction, which gives rise to the transverse sensitivity of $F_{\perp}$ undesirable for bunch transverse dynamics.

2. The head-tail contribution to $F_{\perp}$, which has a sudden jump at the magnet entrance, was not accounted for in the cancellation theory and in other previous 


\section{analyses.}

Actually this head-tail contribution to $F_{\perp}$, which has been included in many previous analyses and numerical simulations, is mainly the head-tail part of $F^{\mathrm{CSCF}}$ in Eq. (4). It is not emphasized in the cancellation study because its effect on the transverse dynamics is cancelled by the potential energy effect.

The inclusion of both tail-head and head-tail interaction in Ref. [3] is indicated in Eq. (8) of Ref. [3], where the limit of integration "refers to front and back of the bunch", rather than being confined only to source particles on the back of the test particle. Similarly, in the analysis of $F_{\perp}$ and its cancellation for a coasting beam, the integrations over ranges of source particles in Eqs. (31) and (33) of Ref. [4] are for angles from 0 to $2 \pi$ (as can be verified), also including the interactions from both front and back of the test particle. More detailed analysis of this head-tail part of $F_{\perp}$ can be found in Eqs. (37) and (38) of Ref. [9].

Note that for two on-orbit particles, the head-tail force given in Eqs. (14) of Ref. [1] is exactly the head-tail (also front-back [8] in this case) part of $F^{\mathrm{CSCF}}$ in Eq. (4) $\left(1 / 4 \pi \epsilon_{0}\right.$ is for unit conversion) for $\beta_{s}=1$ and $r=R$. Using Lienard-Wiechert potential for $A_{s}$ and $\Phi$, one has

$$
F^{\mathrm{CSCF}} \simeq \frac{e^{2}}{R \Delta z} \quad\left(\text { also here }-\frac{e \Phi}{r} \simeq-\frac{e^{2}}{R \Delta z}\right),
$$

with $\Delta z \simeq 2\left|s-s^{\prime}\right|$ the longitudinal distance of the two particles. For the discussions of $F^{\mathrm{CSCF}}$ in Sec. 3.2.2 of Ref. [2], $|\Delta \theta|=\left|s-s^{\prime}\right| / R$ is used, referring to both back-front $\left(s^{\prime} \leq s\right)$ and front-back $\left(s^{\prime} \geq s\right)$ interaction. The sudden jump of $F^{\mathrm{CSCF}}$ at the magnet entrance, as shown in Fig. 1 of Ref. [1] for the example case, was earlier discussed in Sec. 2.2 of Ref. [2] to imply that the "centrifugal space charge force" represents the inertial effect for the potential energy of the test particle due to collective interaction, in the same way as the sudden turn-on of the usual centrifugal force represents the inertial effect of the particle's kinetic energy. The sudden jump for head-tail part of $F_{\perp}$ being different from that for the tail-head part (see Figs. 1 and 2 of Ref. [1]) is because (a) the tail-head part of $F_{\perp}$ has an effective transverse force ( $F_{r}^{\text {eff }}$ of Eq. (8) or Eq. (3)) in addition to $F^{\mathrm{CSCF}}$, and (b) the retardation relation differs for headtail (especially front-back) and tail-head interaction.

One should note that in Eq. (9), similar to $F^{\mathrm{CSCF}}$, the term $e \Phi(t) / r$ also has the head-tail contributions (for example, see Eq. (10)) as well as the tail-head part, and the factor $1 / r$ causes a sudden jump of this term when the test particle enters from a drift $(R=\infty)$ to a circular orbit ( $R$ finite), in a similar way as $F^{\mathrm{CSCF}}$ does. The sensitive dependence of $F_{\perp}$ on the transverse offset of a test particle with respect to the bunch, for both the headtail and tail-head part of $F_{\perp}$ as shown in Figs. 1 and 2 of Ref. [1], is mainly caused by the contribution of local $\left(s^{\prime} \rightarrow s\right.$ and $\left.\left|\mathbf{x}^{\prime}-\mathbf{x}\right| \rightarrow h\right)$ interactions. Since in Eq. (9),
$A_{s}$ and $\Phi$ are dominated by the same singular contributions (see item 4 below) and the sudden jump behavior, the combined effects of $e A_{s}(t) / r$ and $e \Phi(t) / r$ on the transverse dynamics are cancelled in $G_{c}$.

3. Since $\hat{F}_{\perp}$ has a sudden jump at the entrance of a magnet while $\hat{\Phi}$ does not, as shown in Fig. 3 of Ref. [1], there is no effective cancellation between $\hat{F}_{\perp}$ and $\hat{\Phi}$.

The cancellation is between the two terms in $G_{c}$ of Eq. (9), namely, $F^{\mathrm{CSCF}}$ and $e \Phi(t) / r$. It can also be considered as the cancellation between the transverse sensitive part of $F_{\perp}$ and $e \kappa \Phi(t) /(1+\kappa x)$, with the design curvature $\kappa(s)=1 / R(s)$ (more detail see Ref. [2]). The sudden jump of the latter term occurs as the bunch enters from drift $\kappa=0$ to a circular orbit $\kappa=1 / R$. However, in Fig. 3 of Ref. [1], the comparison is between $F_{\perp}$ and $e \Phi / R$ for constant $R$. Thus this comparison incorrectly represents the cancellation for the entrance problem.

4. $G_{c} \simeq 0$ has only been shown by macroparticle simulations.

The analytical argument of $G_{c} \simeq 0$ can be found in Sec. 3.2.2 of Ref. [2] for test particles embedded in a continuous distribution. Let $t$ be an independent variable, and $\mathbf{x}=\mathbf{x}(x, y, s)$ in a Frenet coordinate frame [2] (see Ref. [12] for dynamics formulation using bunch coordinates). For the bunch charge distribution $\rho(\mathbf{x}, t)$ and average velocity distribution $\mathbf{u}(\mathbf{x}, t) \simeq c \mathbf{e}_{s}(s)$, with $\mathbf{e}_{s}\left(s^{\prime}\right) \cdot \mathbf{e}_{s}(s)=\cos \left[\left(s-s^{\prime}\right) / R\right]$, and for $t^{\prime}=$ $t-\left|\mathbf{x}(t)-\mathbf{x}^{\prime}\right| / c$, the (Lorentz gauge) retarded potential terms in Eq. (9) are

$$
\begin{aligned}
& A_{s}(t)=A_{s}(\mathbf{x}(t), t) \\
& \simeq e \int d s^{\prime} d x^{\prime} d y^{\prime} \cos \left(\frac{s^{\prime}-s(t)}{R}\right) \frac{\rho\left(\mathbf{x}^{\prime}, t^{\prime}\right)}{\left|\mathbf{x}^{\prime}-\mathbf{x}(t)\right|} \\
& \Phi(t)=\Phi(\mathbf{x}(t), t) \simeq e \int d s^{\prime} d x^{\prime} d y^{\prime} \frac{\rho\left(\mathbf{x}^{\prime}, t^{\prime}\right)}{\left|\mathbf{x}^{\prime}-\mathbf{x}(t)\right|}
\end{aligned}
$$

Transverse sensitivity arises from local interaction contained in the singular integrand $\rho\left(x^{\prime}, t^{\prime}\right) /\left|\mathbf{x}^{\prime}-\mathbf{x}(t)\right|$ when $\left|s^{\prime}-s(t)\right| \ll 1$, and $\left|\mathbf{x}^{\prime}-\mathbf{x}(t)\right|$ is approximately

$$
\sqrt{\left(s^{\prime}-s(t)\right)^{2}+\left(x^{\prime}-x(t)\right)^{2}+\left(y^{\prime}-y(t)\right)^{2}} .
$$

The two terms in $G_{c}$ (Eq. (9)), $e A_{s} / r$ and $e \Phi / r$, nearly cancel, because they both are dominated by the same local interaction contributions, as can be seen from Eqs. (11) and (12) when $\cos \left[\left(s-s^{\prime}\right) / R\right] \simeq 1$. Since the local contributions to both terms are mainly related to the same bunch charge distribution $\rho(\mathbf{x}, t)$ at $t$, which can be a general bunched beam or a coasting beam, the cancellation applies to a general ultrarelativistic moving charge distribution going through the magnetic bends.

5. Even if $G_{c} \simeq 0$, there will be anyway a strong head-tail contribution in $G_{0}$ that cannot be cancelled away. 
This is a true statement. The term in $G_{0}$ containing the head-tail contributions is $e \Phi(0) / r$ in Eq. (6), and it indeed has sensitive dependence on the test particle's transverse position in the bunch which cannot be cancelled away. However, unlike the centrifugal space charge force $F^{\mathrm{CSCF}}$ (which is cancelled by $e \Phi(t) / r$ in Eq. (9)), this term has no direct impact on the the bunch transverse emittance for an achromatic bending system.

First, it is understood that for a rigid line bunch as studied in Ref. [1, 10], the local contributions to $\Phi(t)$ and to $\Phi(0)$ are about the same. So when $A_{s}(t)$ is cancelled by $\Phi(t)$, there is $\Phi(0)$ added elsewhere; thus there is no obvious cancellation. A clearer picture may be obtained when the bunch evolution over time and particle dynamics advance are taking into account. For example, if the test particle in Figs. 1 and 2 of Ref. [1] is a part of the bunch with $\gamma(t) \simeq \gamma_{0}$, it cannot always remain at the same relative transverse position with respect to the ultrarelativistic rigid bunch.

Second, for an evolving bunch, as the test particle's transverse position within the bunch changes, the transverse force and potential exerted on the particle change with time. The transverse sensitive part of the two terms in Eq. (9), $F^{\mathrm{CSCF}}$ and $e \Phi(t) / r$, cancel, because they have almost the same functional dependence on time (due to the nearly identical local contributions discussed in item 4). If not cancelled, they could potentially cause transverse emittance growth for an acromatic bending system, such as a magnetic chicane in which the charge distribution is longitudinally compressed and transversely stretched in dispersive regions.

Third, unlike $F^{\mathrm{CSCF}}, e \Phi(0)$ in $G_{0}$ is determined fully by $\mathbf{x}(t=0)$ and $\rho\left(\mathbf{x}^{\prime}, t^{\prime}\right)$ for $t^{\prime} \leq 0$. From a simulation point of view, $\Phi(0)$ for each particle needs to be computed only once at the entrance of the beamline of interest. Together $\left[\gamma(0) m c^{2}+e \Phi(0)\right]$ impacts the transverse dynamics as the initial (canonical) energy, where the two terms can be independent from each other. Therefore, even though it has the nearby particle singular contribution (including the head-tail part), $e \Phi(0)$ in Eq. (6) can be considered as a modification to the initial kinetic energy $\gamma(0) m c^{2}$, which indeed has an additional transverse effect in dispersive regions (just as any initial energy offset does), yet to the first order of optics, does not cause transverse emittance growth for a bunch going through an acromatic bending system [7].

Note that in Ref. [4], the equilibrium orbit for each particle is set up using its canonical energy $\gamma\left(R_{e}\right) m c^{2}+$ $e \Phi\left(R_{e}\right)$, and the cancellation discussed in Ref. [4] for a storage ring is referenced to this equilibrium orbit.

6. The cancellation scheme is a regrouping of $F_{\perp}+$ $\Delta E / r$, which can be done to any pair of quantities.

As discussed above, the two cancelled terms in $G_{c}$ (Eq. (9)) both have singular local contributions with head-tail and tail-head parts. If not cancelled, they can potentially cause transverse emittance degradation due to the fact that these terms vary in dispersive regions as the bunch evolves with time. In contrast, the remaining terms have different features: $G_{0}$ depicts the effect of initial canonical energy; $G_{v}$ and $G_{r}$ [11] in Eqs. (7) and (8) mainly depend on the nonlocal back-front interaction, with some residual transverse sensitivity which is different in magnitude and behavior compared to the two terms which are cancelled.

\section{SUMMARY}

As remarked in Ref. [2], when the self-consistent dynamics is treated with both the transverse and longitudinal interactions taken into account, the canonical formulation, which explicitly reveals the cancellation effect, is completely equivalent to the Lorentz force approach with the cancellation implicitly taken care of. Here Trafic ${ }^{4}$ is a good example that one does not need the cancellation effect to solve realistic problems [1]. However, in the CSR study, due to the complexity of the problem, sometimes one effect (such as the head-tail part of $F_{\perp}$ ) is emphasized without taking into account the other effect (such as the head-tail part of $e \Phi(t) / r$ ) which is strongly correlated to the former one). Viewing the interaction process from a different angle may sometimes be instructive in this regard, which also allows further analysis [12] and simulations. The cancellation theory shows that the transverse emittance is related to the change of kinetic energy and the transverse force. Since the time dependent parts of the singular local contributions to these two terms are oppositely correlated, after reduction, only the effective forces have impact on the transverse emittance growth.

\section{REFERENCES}

[1] G. Geloni, E. Saldin, E. Schneidmiller and M. Yurkov, DESY 03-165, 2003.

[2] R. Li and Ya. S. Derbenev, JLAB-TN-02-054, 2002.

[3] R. Talman, PRL, vol.56, p1429, 1986.

[4] E. P. Lee, Particle Accelerators, 25, 241 (1990).

[5] Ya. S. Derbenev and V. D. Shiltsev, SLAC-Pub-7181, 1996.

[6] R. Li, Proceedings of the 2nd ICFA Advanced Accelerator Workshop, p.369, Los Angeles, 1999.

[7] R. Li, Proceedings of 2002 EPAC, Paris (2002).

[8] Note that in this paper, the head-tail interaction refers to $z^{\prime}>z$, with $z\left(z^{\prime}\right)$ the longitudinal position of the test (source) particle in the bunch; while the front-back interaction refers to $s^{\prime}>s$, with $s\left(s^{\prime}\right)$ the pathlength position for the test (retarded) particle. For cases with bunch tilt, see [12].

[9] G. V. Stupakov, Proceedings of the Nonlinear and Collective Phenomena in Beam Physics Workshop, pg.334, 1998.

[10] G. Geloni et al., DESY 02-48 and DESY 03-44, (2002).

[11] The term $e d A_{r} / c d t$ in $G_{r}$ (Eq. (8)) should be considered jointly with $m \dot{x} d \gamma / d t$ on the left side of Eq. 1 (see [12]).

[12] R. Li, JLAB-TN-05-046, 2005. 